\title{
Multistep Prediction of Physiological Tremor for Surgical Robotics Applications
}

\author{
Kalyana C. Veluvolu*, Senior Member, IEEE, Sivanagaraja Tatinati, Sun-Mog Hong, Member, IEEE, \\ and Wei Tech Ang, Member, IEEE
}

\begin{abstract}
Accurate canceling of physiological tremor is extremely important in robotics-assisted surgical instruments/procedures. The performance of robotics-based hand-held surgical devices degrades in real time due to the presence of phase delay in sensors (hardware) and filtering (software) processes. Effective tremor compensation requires zero-phase lag in filtering process so that the filtered tremor signal can be used to regenerate an opposing motion in real time. Delay as small as $\mathbf{2 0} \mathrm{ms}$ degrades the performance of human-machine interference. To overcome this phase delay, we employ multistep prediction in this paper. Combined with the existing tremor estimation methods, the procedure improves the overall accuracy by $60 \%$ for tremor estimation compared to single-step prediction methods in the presence of phase delay. Experimental results with developed methods for 1-DOF tremor estimation highlight the improvement.
\end{abstract}

Index Terms-Autoregressive (AR), band limited multiple linear Fourier combiner (BMFLC), inertial sensors, Kalman filter, multistep prediction, physiological motion, tremor.

\section{INTRODUCTION}

$\mathbf{R}$ OBOTICS-ASSISTED surgical instruments/procedures are increasingly playing an important role for biological motion compensation [1]-[3] due to their robustness, high precision, and estimation accuracy [4]. With the aid of this robotic technology, significant research was focused on compensation of various biological motions like heart beat in intracardiac surgery [1], [5], respiratory motion in treatment of lung tumor [2], pathological tremor [6], and physiological tremor [3], [7]-[10].

Physiological tremor exists in all humans with amplitude ranges between 50 and $100 \mu \mathrm{m}$ in each principal axis and lies

Manuscript received December 19, 2012; revised March 24, 2013; accepted May 9, 2013. Date of publication June 12, 2013; date of current version October 16, 2013. This work was supported by the Basic Science Research Program through the National Research Foundation of Korea funded by the Ministry of Education, Science and Technology Grants under 2011-0023999 and 20110013475). Asterisk indicates corresponding author.

${ }^{*} \mathrm{~K}$. C. Veluvolu is with the School of Electronics Engineering, College of IT Engineering, Kyungpook National University, Daegu 702-701, Korea (e-mail: veluvolu@ee.knu.ac.kr).

S. Tatinati and S.-M. Hong are with the School of Electronics Engineering, College of IT Engineering, Kyungpook National University, Daegu 702-701, Korea (e-mail: tatinati@ee.knu.ac.kr; smhong@ee.knu.ac.kr).

W. T. Ang is with the School of Mechanical and Aerospace Engineering, Nanyang Technological University, Singapore 639798 (e-mail: wtang@ ntu.edu.sg).

Color versions of one or more of the figures in this paper are available online at http://ieeexplore.ieee.org.

Digital Object Identifier 10.1109/TBME.2013.2264546 in the frequency range of $8-12 \mathrm{~Hz}$ [11], [12]. This tremor leads to an intolerable imprecision of the surgical procedure (e.g., vitroretinal surgery) which requires a positioning accuracy of about $10 \mu \mathrm{m}$ [13]. The high level of manual accuracy demanded by microsurgery restricts the number of qualified surgeons. Significant amount of research has been conducted on tremor characteristics (pathological and physiological tremors) and its real-time compensation with robotic procedures [6]-[8], [14]-[17]. To retain the advantages possessed by the human surgeons and to augment tip positioning accuracy, hand-held robotic instruments were developed for compensation of physiological tremor in real time [9], [14], [18]. In these instruments, filtering plays a vital role in attaining high accuracy. The filtered tremor signal from the sensed motion is used to generate an opposing motion to compensate for the tremor motion in real time. For effective tremor compensation, zero-phase lag is required in the filtering process. Although linear filters are successful in filtering the tremor, they introduce phase delay into the process [19]. In [20], it was shown that delay as small as $30 \mathrm{~ms}$ may degrade the performance in human-machine control applications.

To overcome the inherent disadvantages of linear filters, several adaptive algorithms are developed. In [7], [14], and [15], Fourier-series-based adaptive algorithms named as weighted frequency Fourier linear combiner (WFLC) and band limited multiple linear Fourier combiner (BMFLC) are proposed. In [21]-[23], the autoregressive (AR)-method-based tremor estimation methods are proposed. Comparative performance of all zero-phase physiological tremor estimation methods can be found in [7].

In robotics-assisted hand-held instruments, accelerometers form the core part for tremor sensing due to its small size and versatility [9]. Although adaptive tremor estimation introduces no additional phase delay, real-time tremor compensation accuracy depends on several factors such as prefiltering, numerical integration, noise, jerk, and drift. It will be later shown that a phase delay of 16-20 ms is unavoidable for tremor compensation and this delay adversely effects the physiological tremor cancellation.

Multistep prediction is popular where time delay is inevitable or posteriori information is required [24]. Multistep prediction has been successfully applied for several physiological motion predictions [1], [23], [25] and time series forecasting [26], [27]. For compensation of heart beat in intracardiac surgery, multistep prediction was employed to avoid latency of $90 \mathrm{~ms}$ in the procedure [1], [5]. In [1], WFLC with extended Kalman filtering (EKF) was employed for multistep prediction of heart beat. 


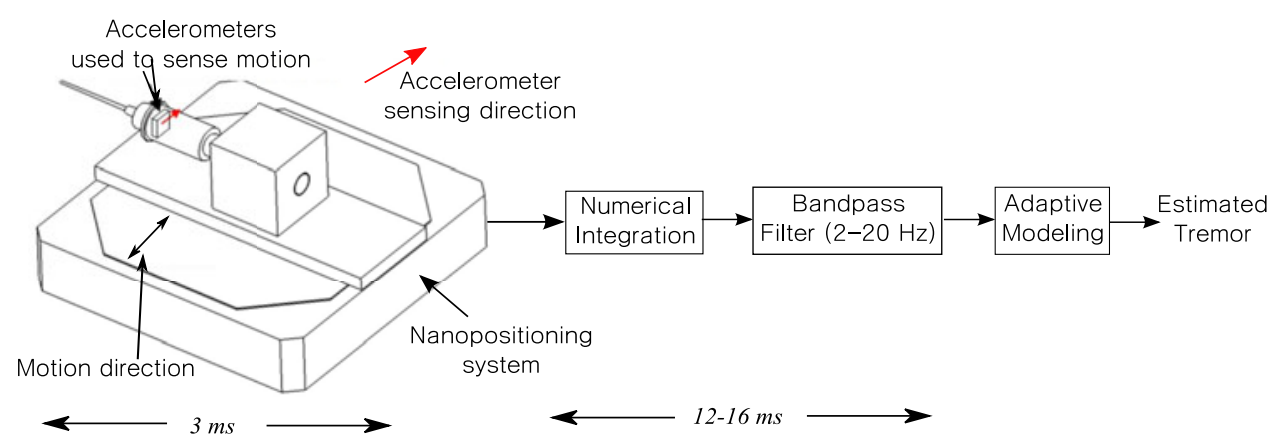

Fig. 1. Block diagram for real-time implementation.

Adaptive radiotherapy requires the respiratory motion prediction to track and compensate for lung tumor [2], [25]. Recently, in [23], multistep prediction was employed for pathological tremor estimation based on EKF. Physiological tremor prediction is more challenging due to its low amplitude and highfrequency range $(8-12 \mathrm{~Hz})$ compared to respiratory and cardiac motions that have low-frequency range $(<1 \mathrm{~Hz})$.

In this paper, we analyze the suitability of multistep prediction for real-time tremor estimation. Two existing methods BMFLC and AR are modified to develop multistep prediction methods. A study was conducted with the tremor data of five surgeons and five novice subjects for various prediction lengths and various sampling frequencies. Several variants of these methods are reviewed to check the suitability for real-time tremor compensation. The phase delay involved in the real-time tremor cancellation is analyzed. Experimental results for 1-DOF tremor estimation in the presence of delay show good improvement compared to earlier methods.

The rest of the paper is organized as follows: In Section II, tremor data collection and experimental setup employed are first discussed. Later multistep-based methods are discussed. Section III presents simulation and experimental results. Discussions are provided in Section IV. Section V concludes the paper.

\section{METHODS}

In this section, we first discuss about the physiological tremor data collection. The experimental setup employed for evaluation of the method is also discussed together with the delay characteristics. Later, multistep prediction of physiological tremor with BMFLC- and AR-based methods is discussed.

\section{A. Physiological Tremor Data}

Tremor recordings are performed through the micro-motion sensing system $\left(M^{2} S^{2}\right)$. The resolution, minimum accuracy, and sampling rate of the $M^{2} S^{2}$ are $0.7 \mu \mathrm{m}, 98 \%$, and $250 \mathrm{~Hz}$, respectively [28]. Several surgeons and novice subjects participated in this study. All subjects gave informed consent prior to the test and reported no physical or cognitive impairments. The subjects had their wrists rested on a small platform of the $\left(M^{2} S^{2}\right)$ and were asked to take a comfortable seating position. They had to hold the stylus between their index finger and thumb in order to ensure that all subjects have similar grip across trials. Two types of tasks are performed by subjects.

1) Stationary Task: In this task, subjects are instructed to point the laser light at the center point of the platform with the stylus provided for $30 \mathrm{~s}$ duration.

2) Tracing Task: In this task, subjects trace the circumference of a circular path on the platform for $30 \mathrm{~s}$, with the speed that is realistic for surgical micromanipulation tasks.

Several trials were conducted with all the subjects under different magnification and force conditions. Subjects performed two trials for each different setting with approximately $1 \mathrm{~min}$ break in between settings. Sampling rate of $500 \mathrm{~Hz}$ is employed. For more information about magnification and force conditions, see [29]. Data of five healthy subjects and five surgeons, and six trial data per subject are considered for analysis in this paper.

\section{B. Experimental Setup and Latency}

The block diagram representation for experimental setup and filtering is shown in Fig. 1. Accelerometers (ADXL 203, Ana$\log$ devices, USA) are employed to sense the motion. The tremor-like motion is generated using a commercially available nanopositioning stage (P-561.3CD from Physik Instrumente, Germany) on which a physiological tremor compensation instrument (ITrem [28]) consisting of accelerometers is mounted. For more information about placing accelerometers in the robotics instrument, see [28].

The nanopositioning stage is driven in one axis to replicate the tremor motion within $100 \mathrm{~nm}$, the maximum range of the stage. The orientation and accelerometer along with the direction motion are shown in the schematic diagram (see Fig. 1). The voltage output from the accelerometer is acquired at $500 \mathrm{~Hz}$ using a 16-bit data acquisition card (PD2-MF-150, United Electronic Industries, Inc, USA). QNX operating system is employed for real-time implementation. Before the experiment, static calibration of the accelerometer is performed using a gravity value of $9.81 \mathrm{~m} / \mathrm{s}^{2}$. The sensitivity value of the accelerometer obtained from the calibration is $980 \mathrm{mV} / \mathrm{g}$. The voltage readings acquired from accelerometer are converted to acceleration with a quadratic function. Furthermore, the gravitational component is subtracted from the measured acceleration.

The accelerometer contains an on-board low-pass filter; the hardware filter time constant calculated from the step-response is approximately $3 \mathrm{~ms}$. Numerical integration is performed for 


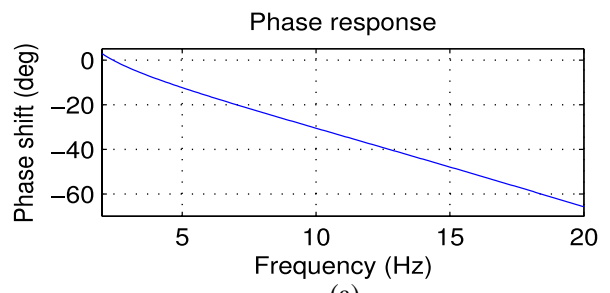

(a)

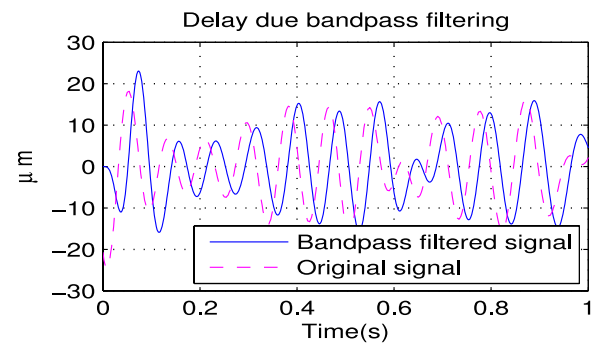

(b)

Fig. 2. Frequency response of fifth-order Butterworth bandpass filter. (a) Phase response. (b) Input tremor signal (solid line) and delayed tremor signal due to bandpass filtering (dotted line).

TABLE I

Performance of BMFlC-KF [7] In the Presence of Phase Delay

\begin{tabular}{cc}
\hline Phase delay $(m s)$ & Estimation accuracy \\
\hline $0 \mathrm{~ms}$ & 99.96 \\
$4 \mathrm{~ms}$ & 78.33 \\
$8 \mathrm{~ms}$ & 56.37 \\
$16 \mathrm{~ms}$ & 15.53 \\
$20 \mathrm{~ms}$ & 7.16 \\
\hline
\end{tabular}

converting acceleration to position. After this stage, to separate the tremulous motion from the voluntary motion and to remove the unwanted integration drift and noise [30], a fifth-order Butterworth filter with passband $2-20 \mathrm{~Hz}$ is employed as shown in the block diagram. The filter order and cutoff frequency need to be chosen so that the filter removes unwanted low-frequency drift significantly. The choice depends on the sensor noise level and the frequency spectrum of the tremor signal.

This filtering stage is the main source for phase delay in real time. For illustration, phase response of fifth-order Butterworth bandpass filter with passband $2-20 \mathrm{~Hz}$ is shown in Fig. 2(a). To highlight the phase delay, a bandpass-filtered tremor signal and an original signal are shown in Fig. 2(b). Phase delay is frequency dependent and the actual phase delay depends on tremor frequency characteristics. As dominant frequency of tremor lies within the range of $8-12 \mathrm{~Hz}$, an average $12-16 \mathrm{~ms}$ phase delay is identified. Piezo-electric actuators are employed to generate the tremor signal in opposite direction for active cancellation and this stage requires $1 \mathrm{~ms}$. Thus, a total of $16-20 \mathrm{~ms}$ phase delay is unavoidable due to hardware and software filtering as shown in Fig. 1.

In order to study the effect of phase delay on estimation accuracy, various delays are incorporated into the BMFLC-KF method. Estimation accuracy for various phase delays with single-step prediction is tabulated in Table I. To quantify the performance, in this paper, we employ the root mean square (RMS) defined as $\operatorname{RMS}(s)=\sqrt{\left(\sum_{k=1}^{k=m}\left(s_{k}\right)^{2} / m\right)}$, where $m$ is

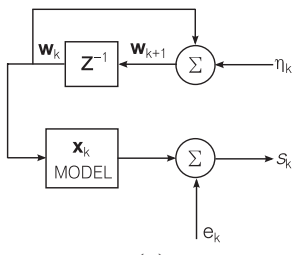

(a)

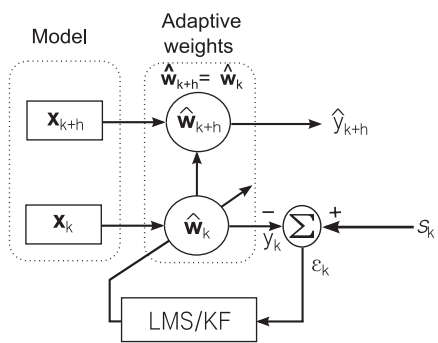

(b)
Fig. 3. (a) Signal model. (b) Block diagram for multistep prediction.

the number of samples and $s_{k}$ is the input signal at instant $k$. Based on RMS, \%Accuracy is defined as

$$
\% \text { Accuracy }=\frac{\operatorname{RMS}(s)-\operatorname{RMS}(e)}{\operatorname{RMS}(s)} \times 100
$$

where $e$ is the prediction error between an actual signal and a predicted signal.

It can be seen that a delay as small as 16-20 ms can adversely affect the performance of the robotic instrument in canceling the tremor in real time. To overcome this phase delay, multistep prediction based on BMFLC and AR methods is developed in this paper for tremor estimation.

\section{Multistep Prediction With BMFLC (MS-BMFLC)}

To overcome the problems with tremor signals comprising of multiple dominant frequencies, BMFLC [7] was recently developed. To estimate the tremor signal in the predefined band $\left[\omega_{1}-\omega_{n}\right]$, a series comprising of sine and cosine components are combined to form BMFLC

$$
y_{k}=\sum_{r=1}^{n} a_{r k} \sin \left(\omega_{r} k\right)+b_{r k} \cos \left(\omega_{r} k\right)
$$

where $y_{k}$ denotes the estimated signal at sampling instant $k$. $a_{r k}$ and $b_{r k}$ represent the adaptive weights corresponding to the frequency $\omega_{r}$ at instant $k . \Delta \omega$ represents the step size in the frequency band $\left[\omega_{1}-\omega_{n}\right]$ division and $n=\left|\omega_{1}-\omega_{n}\right| / \Delta \omega$. The series only considers " $n$ " fundamental frequencies in the band.

The signal model employed for adaptive estimation is shown in Fig. 3(a). $s_{k}$ represents the amplitude of the signal at the $k$ th sample. The model $\mathbf{x}_{k}$ together with the adaptive weights $\mathbf{w}_{k}$ represents the time-varying model for the signal $s_{k}$ in the state-space form as

$$
\begin{aligned}
s_{k} & =\mathbf{w}_{k}^{T} \mathbf{x}_{k}+e_{k} \\
\mathbf{w}_{k+1} & =\mathbf{w}_{k}+\eta_{k}
\end{aligned}
$$

where $e_{k}$ and $\eta_{k}$ are measurement noise and process noise, respectively. The state-space representation for BMFLC can be described in the form

$$
\mathbf{x}_{k}=\left[\begin{array}{ccc}
{\left[\sin \left(\omega_{1} k\right)\right.} & \cdots & \left.\sin \left(\omega_{n} k\right)\right]^{T} \\
{\left[\cos \left(\omega_{1} k\right)\right.} & \cdots & \left.\cos \left(\omega_{n} k\right)\right]^{T}
\end{array}\right]
$$


TABLE II

ADAPTATION SCHEMES

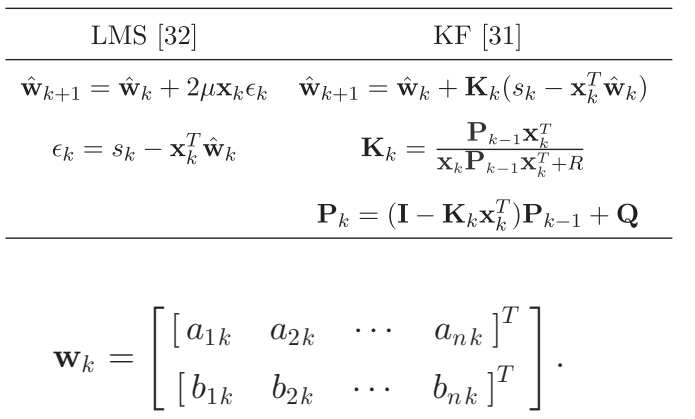

When no priori information is available, the state dynamics can be best described by a random walk model (3) [31]. Adaptive algorithms like least mean square (LMS) and Kalman filter (KF) can be employed for adaptive estimation of state $\mathbf{w}_{k}$. For amplitude adaptation with LMS, the adaptation scheme is provided in Table II. $\mu$ is the adaptive gain parameter that controls the adaptation rate [32] for estimation of $\hat{\mathbf{w}}_{k}$. With the assumption that $e_{k}$ and $\eta_{k}$ are uncorrelated, zero-mean, Gaussian white noise processes with covariances $\mathbf{R}$ and $\mathbf{Q}$. The estimates for state $\hat{\mathbf{w}}_{k}$ and the state error covariance $\mathbf{P}_{k}$ can be obtained recursively by KF [33] with proper initial conditions $\mathbf{w}_{0}$ and $\mathbf{P}_{0}$. The KF adaptation scheme is provided in Table II. $\mathbf{K}_{k}$ is the Kalman gain vector. Although the premise of the noise may not hold for the signal, the KF can provide the minimum meansquared error estimators within the class of linear estimators. Employing LMS or KF, the estimated output $y_{k}$ and prediction error $\epsilon_{k}$ [shown in Fig. 3(b)] can be obtained as

$$
\begin{aligned}
y_{k} & =\hat{\mathbf{w}}_{k}^{T} \mathbf{x}_{k} \\
\epsilon_{k} & =s_{k}-y_{k} .
\end{aligned}
$$

In contrast to aforementioned, multistep prediction requires prediction of the signal $y_{k+h}$ several samples ahead (say $h$ samples), based on its past observations $s_{k}, s_{k-1} \ldots$, as shown in Fig. 3(b). The model is represented by the reference vector, and the weights $\left(\hat{\mathbf{w}}_{k}\right)$ represent the estimated adaptive parameters (states). In BMFLC, the reference vector $\left(\mathbf{x}_{k}\right)$ comprises sine and cosine components of predefined input frequencies. Hence, the input reference can be accurately known for prediction. Assuming adaptive weights to remain constant over prediction length, an accurate multistep prediction can be obtained. With the reference vector $\left(\mathbf{x}_{k+h}\right)$ accurately known at the time instant $k+h$, the estimated parameters (weights) at the current sample $\left(\hat{\mathbf{w}}_{k}\right)$ can be employed to obtain multistep prediction for output $y_{k+h}$ as

$$
\hat{y}_{k+h}=\hat{\mathbf{w}}_{k}^{T} \mathbf{x}_{k+h} \text {. }
$$

The model parameters of BMFLC used for the estimation of tremor are amplitude weights $\left(\mathbf{w}_{k}\right)$ and the reference vector $\left(\mathbf{x}_{k}\right)$. The block diagram representation for multistep prediction with BMFLC is shown in Fig. 4. The amplitude of the signal $h$ samples ahead can be predicted as

$$
\hat{y}_{k+h}=\mathbf{x}_{k+h}^{T} \hat{\mathbf{w}}_{k+h}
$$

where

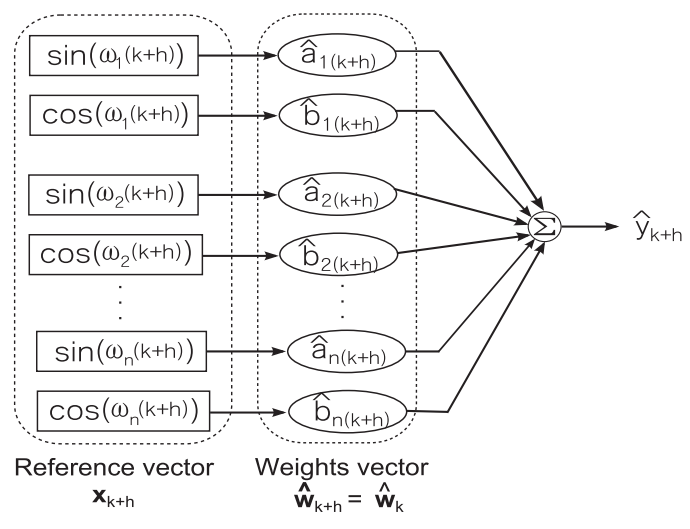

Fig. 4. Multistep prediction with BMFLC.



Fig. 5. Multistep prediction with the AR method.

1) $\hat{\mathbf{w}}_{k+h}=\hat{\mathbf{w}}_{k}$ (amplitude weight vector (state vector) remains constant for $k$ to $k+h$ samples);

2) $\mathbf{x}_{k+h}=\left[\begin{array}{c}{\left[\sin \left(\omega_{1}(k+h)\right) \cdots \sin \left(\omega_{n}(k+h)\right)\right]^{T}} \\ {\left[\cos \left(\omega_{1}(k+h)\right) \cdots \cos \left(\omega_{n}(k+h)\right)\right]^{T}}\end{array}\right]$.

Hereafter, multistep prediction-based BMFLC-LMS and BMFLC-KF are referred as MS-BMFLC-LMS and MSBMFLC-KF, respectively.

\section{Multistep Prediction With the AR model (MS-AR)}

The AR model is a type of random process which is popular for prediction of various types of natural phenomena. It is also one of the linear prediction methods that is designed to predict an output of a system based on the previous outputs. The AR model of order $M$ can be represented as $\operatorname{AR}(M)$, described as

$$
s_{k}=\sum_{i=1}^{M} w_{i} s_{k-i} \text {. }
$$

By denoting

$$
\begin{aligned}
\mathbf{w}_{k} & =\left[\begin{array}{llll}
-w_{1} & -w_{2} & \cdots & -w_{M}
\end{array}\right]^{T} \\
\mathbf{x}_{k} & =\left[\begin{array}{llll}
s_{k-1} & s_{k-2} & \cdots & s_{k-M}
\end{array}\right]
\end{aligned}
$$

the AR model can be represented in the state-space form (2), (3). Adaptive algorithms both LMS and KF can be employed as discussed in the previous section.

The AR model prediction is dependent on the delayed input vector $\mathbf{x}_{k}$ and amplitude weights $\mathbf{w}_{k}$. Multistep prediction scheme with the AR model is shown in Fig. 5. The amplitude of the signal predicted at $h$ samples ahead using the AR model 


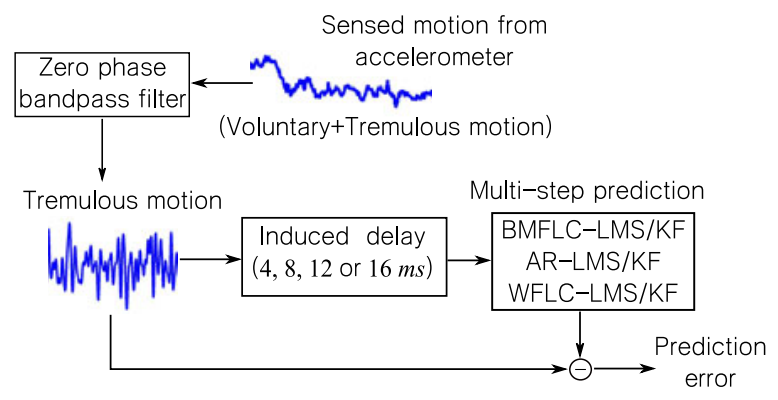

Fig. 6. Performance analysis.

can be obtained as

$$
\hat{y}_{k+h}=\hat{\mathbf{x}}_{k+h}^{T} \hat{\mathbf{w}}_{k+h}
$$

where

1) $\hat{\mathbf{w}}_{k+h}=\hat{\mathbf{w}}_{k}$ (the amplitude weight vector remains constant for $k$ to $(k+h)$ samples);

2) $\hat{\mathbf{x}}_{k+l}=\left[\hat{y}_{k-l-1} \hat{y}_{k-l-2} \cdots \hat{y}_{k-l-M}\right] ; l=1,2, \cdots, h$ (the input vector $\hat{\mathbf{x}}_{k}$ is updated iteratively).

\section{RESULTS}

In this section, we analyze the performance of the proposed multistep prediction methods through simulations and experiments. To quantify the performance, we employ \%Accuracy as defined in (1).

\section{A. Performance Analysis}

In this section, we present performance analysis of all methods through simulations for the data of ten subjects. We also include WFLC-LMS/KF methods for comparison. To accurately analyze the performance in the presence of delay, we induce a known delay into the process as shown in Fig. 6. A zero-phase bandpass filter is employed to remove the voluntary motion. We then analyze the comparative performance of all methods for various prediction lengths and various sampling rates.

1) Parameter Selection: For adaptive estimation algorithms, parameter selection and initialization can affect the estimation accuracy. Parameter selection for BMFLC- and WFLC-based methods is well documented. For more information, see [3], [7], and [15]. For the AR model, optimal order selection and initialization depends on data characteristics. For the tremor data collected in our study, Akakie information criterion (AIC) [34] identified the third-order of the AR model as the optimal order. Furthermore, to reduce the initial adaptation period required for the weights of AR methods, analysis was conducted on steadystate weights for all the subjects. Results show that the variance of the steady-state weights across all subjects and tasks is negligible. For the sake of initialization, we select a common set of filter coefficients as $\mathbf{w}_{0}=\{-2.88,-0.94,2.83\}$ to initialize both the methods AR-LMS and AR-KF. Parameter selection and initialization for all methods are tabulated in Table III.

2) Estimation Accuracy Versus Prediction Length: We choose various prediction lengths [4 (2 samples), 8, 16, and $20 \mathrm{~ms}$ (ten samples)] for the tremor signal to analyze the performance of all methods. To quantify the performance, analysis is conducted on the tremor data of ten subjects (five surgeons and five subjects) with six trials/subject. The statistical results (mean and variance) obtained for all the methods are shown in Fig. 7. Results for different prediction lengths $(4,8,16$, and $20 \mathrm{~ms})$ are shown together with single-step prediction for comparison.

For all prediction lengths, KF-based methods outperform its LMS counterparts. For single-step prediction methods \%Accuracy decreases as prediction length increases. With the multistep prediction method, a good estimation accuracy can be obtained for higher prediction lengths as shown in Fig. 7. For example, the estimation accuracy obtained with AR-KF is $8 \pm 3 \%$ for $20 \mathrm{~ms}$ ahead prediction, whereas with MS-AR-KF the estimation accuracy increases to $81 \pm 2 \%$. MS-AR-KF and MS-BMFLC-KF perform better than MS-WFLC-KF for higher prediction lengths.

For illustration, the prediction errors for all methods for $20 \mathrm{~ms}$ (ten samples) of prediction length for Subject \#3 (tracing task) are shown in Fig. 8. The tremor signal and the prediction error obtained with BMFLC-KF for $20 \mathrm{~ms}$ is shown in Fig. 8(a) and (b), respectively. The results obtained with multistep prediction methods (MS-WFLC-KF, MS-BMFLC-KF, and MS-AR-KF) are shown in Fig. 8(c)-(e). This clearly highlights the robustness and suitability of the multistep prediction methods for tremor prediction.

3) Estimation Accuracy Versus Sampling Rate: The number of samples required for prediction is proportional to the sampling frequency employed; this indeed affects the prediction accuracy for high sampling frequencies. The optimal order for the AR model is identified with AIC for different sampling frequencies. With $1000 \mathrm{~Hz}$ sampling frequency, AIC identified 32-order as the optimal order for 16 and $20 \mathrm{~ms}$ prediction lengths. For the rest of prediction lengths (4 and $8 \mathrm{~ms}$ ), third order is identified as optimal order for the AR model. Prediction accuracies obtained for various sampling frequencies for all multistep tremor estimation methods are shown in Fig. 9. MS-BMFLC-KF accuracy remained constant irrespective of the sampling rate. For the case of the MS-AR-KF method, estimation accuracy decreased with increase of the sampling frequency.

4) Computational Complexity: For real-time tremor compensation in robotics instruments, computational complexity also plays an important role in minimizing the delay. The computational complexity of adaptive algorithms mainly relies on observation and state-space dimensions of the method [35]. The computational complexity of KF is given by $O\left(3 l n^{2}\right) ; l$ and $n$ are observation and state dimensions, respectively [35]. As error is a scalar quantity, the computational complexity of LMS is given by $O(N)$, where $N$ is the order of the filter. For the case of WFLC and AR, prefiltering is required to remove the voluntary component in tremor. For real-time implementation of a Butterworth $N$ th-order filter, the number of operations required is $2(2 N+1)$. Thus, a fifth-order filter will require 22 operations. A comparative study is conducted on all the methods for computational complexity and the number of operations is tabulated in Table IV. BMFLC-KF requires more operations compared to other methods, whereas AR requires less number of operations. Depending on the available memory and computational power, the suitable method can be selected for real-time applications. 
TABLE III

METHODS AND PARAMETERS

\begin{tabular}{ll}
\hline Method & Model parameters and initial conditions \\
\hline WFLC-LMS [14] & $\mu_{0}=1.10^{-5} ; \mu=5.10^{-4} ; f_{0}=7 \mathrm{~Hz} ;$ \\
WFLC-KF [15] & $f_{0}=7 \mathrm{~Hz} ; \mu_{0}=1.10^{-5} ; \mu_{1}=5.10^{-4} ; M=1 ; R=0.01 ; \mathbf{Q}=0.01 \times \mathbf{I} ; \mathbf{P}_{0}=0.01 \times \mathbf{I} ;$ \\
BMFLC-LMS [7] & $\omega_{1}=2 \pi \times 7 ; \omega_{n}=2 \pi \times 14 ; \Delta \omega=0.1 ; \mu=0.01 ;$ \\
BMFLC-KF [7] & $\omega_{1}-\omega_{n}=7-14 \mathrm{~Hz} ; \Delta=0.1 ; R=0.01 ; \mathbf{Q}=0.01 \times \mathbf{I} ; \mathbf{P}_{0}=0.01 \times \mathbf{I} ;$ \\
AR-LMS & $\mu=0.5 ; M=3 ; \mathbf{w}_{0}=[-2.88,2.83,-0.94] ;$ \\
AR-KF & $M=3 ; \mathbf{w}_{0}=[-2.88,2.83,-0.94] ; R=0.001 ; \mathbf{Q}=0.01 \times \mathbf{I} ; \mathbf{P}_{0}=0.001 \times \mathbf{I} ;$ \\
\hline
\end{tabular}



(a)



(b)

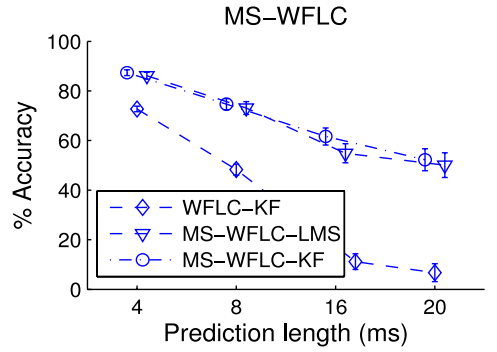

(c)

Fig. 7. Multistep prediction for various prediction lengths: (a) xBMFLC, (b) AR, and (c) WFLC. Representation is standard deviation around mean.

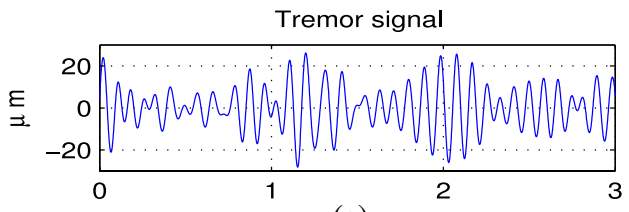

(a)

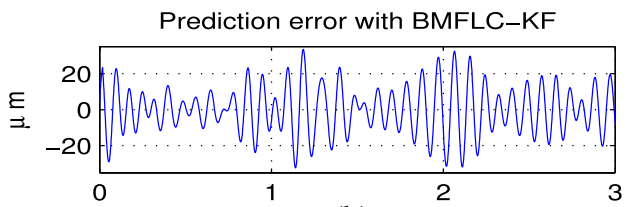

(b)

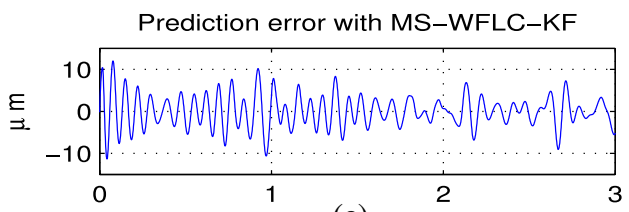

(c)


(e)

Fig. 8. (a) Tremor signal (Subject \#3, tracing task). (b) Prediction error with single-step prediction for BMFLC-KF. (c)-(e) Prediction error during the multistep prediction with $20 \mathrm{~ms}$ (ten samples) of prediction length for WFLC-KF, BMFLC-KF, and AR-KF.

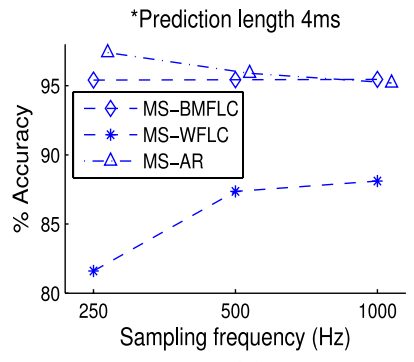

(a)

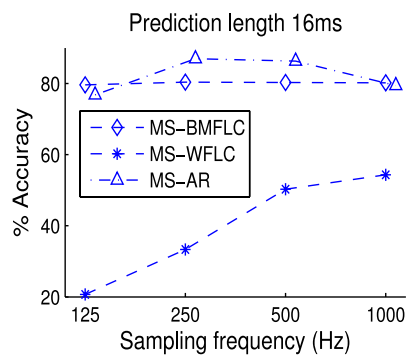

(c)

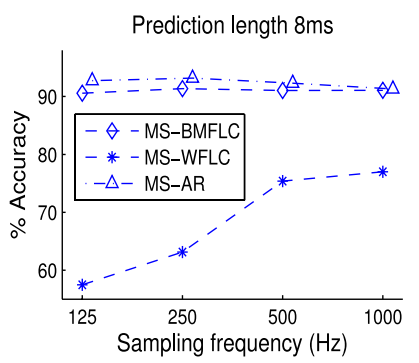

(b)

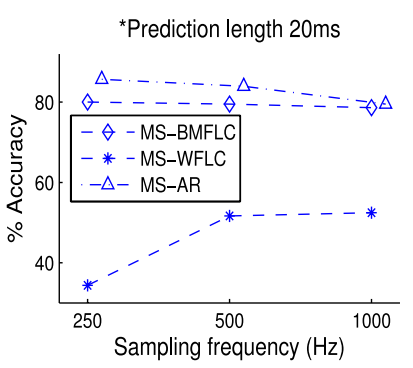

(d)
Fig. 9. Multistep prediction accuracy for various sampling frequencies and various prediction lengths. (a) Prediction length $4 \mathrm{~ms}$. (b) Prediction length $8 \mathrm{~ms}$. (c) Prediction length $16 \mathrm{~ms}$. (d) Prediction length $20 \mathrm{~ms}$.

TABLE IV

COMPUTATIONAL COMPLEXITY

\begin{tabular}{llc}
\hline Method & Parameters & Operations $(\mathrm{O}(\cdot))$ \\
\hline WFLC [14] & $n=4$ & 26 \\
WFLC-KF [15] & $n=4$ & 122 \\
BMFLC-LMS [7] & $\Delta \omega=0.1, n=160$ & 160 \\
BMFLC- KF [7] & $\Delta \omega=0.1, n=160$ & 76800 \\
AR- LMS & $n=3$ & 25 \\
AR-KF & $n=3$ & 52 \\
\hline
\end{tabular}

\section{B. Experimental Validation}

In this section, we present the experimental results for MS-AR-KF and MS-AR-LMS methods. Since BMFLC was 


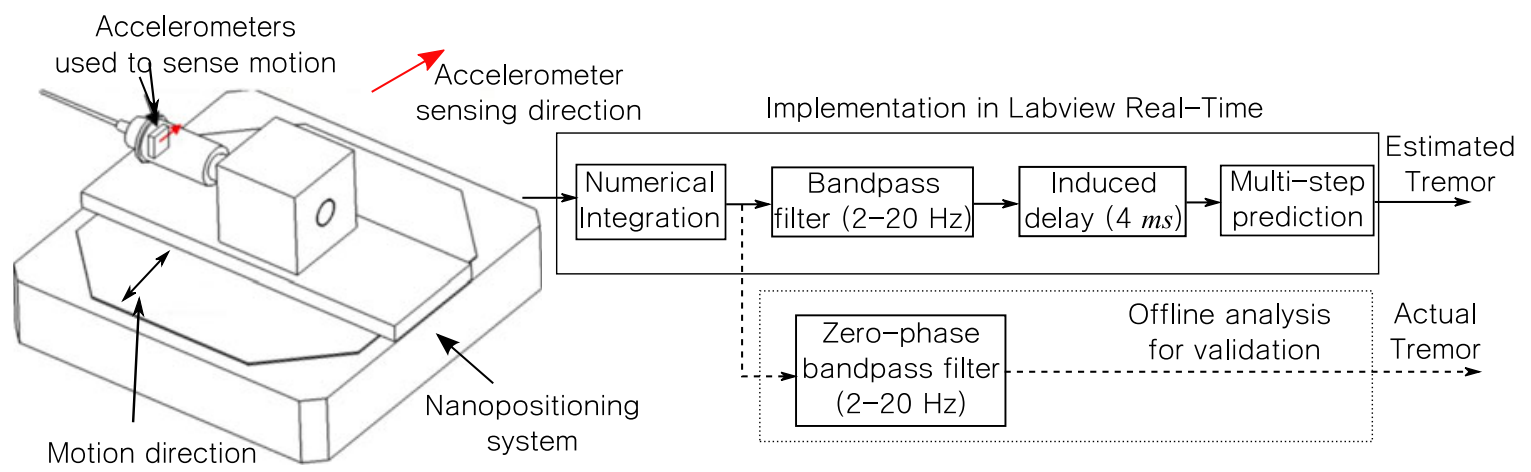

Fig. 10. Experimental procedure.

evaluated experimentally earlier [36] and as the performance was similar to AR-based methods, we only evaluate AR methods experimentally in this section. In order to simulate the realtime estimation/cancellation scenario, we employ the procedure shown in Fig. 10 for experimental validation. To include the delay caused by hardware low-pass filter at the beginning and delay incurred at the cancellation end, a $4 \mathrm{~ms}$ delay is induced into the procedure as shown in Fig. 10. To obtain the ground truth for performance validation, we employ zero-phase bandpass filter in offline. A zero-phase bandpass filter processes the data in bidirections to remove the phase delay involved. As such, this procedure can only be used for offline analysis.

As discussed earlier in Section II-B, the nanopositioning stage is driven in one axis to replicate the tremor motion from the subject as shown in Fig. 10. Experiments are conducted with data of three subjects [S\#1 (tracing task), S\#2 (tracing task), and S\#4 (pointing task)] with two trials per subject. Parameters and initial conditions for real-time experiments are similar to simulation experiments.

For illustration, results obtained with the tremor data of subject \#1 (tracing task) are shown in Fig. 11. The zero-phase bandpass-filtered tremor signal is shown in Fig. 11(a). With the bandpass filter stage employed, an average phase delay in the range of $14-16 \mathrm{~ms}$ is introduced depending on the tremor characteristics. Together with the induced delay $4 \mathrm{~ms}$, ten samples ahead prediction (20 ms) is considered. With MS-AR-LMS and MS-AR-KF prediction methods, accuracies obtained are $51 \pm 2 \%$ and $66 \pm 1 \%$, respectively. For the sake of comparison, a single-step prediction error with AR-KF is shown in Fig. 11(b). Due to $20 \mathrm{~ms}$ phase delay, the \%Accuracy for singlestep prediction with AR-KF is $7 \pm 1 \%$. The results obtained for all the three subjects show similar performance. The $\%$ accuracies obtained with MS-AR-LMS and MS-AR-KF methods are $53 \pm 4 \%$ and $64 \pm 2 \%$, respectively. Experimental results show that MS-AR-KF improves prediction accuracy by over $60 \%$ compared to single-step prediction. Although 1-DOF cancellation is discussed in this paper, the proposed method can be applied for the three axes separately and 3-DOF cancellation of tremor can be achieved simultaneously.

\section{DisCUSSIONS}

For separation of the voluntary motion and tremor motion, a bandpass filter is employed in this paper. Although a bandpass

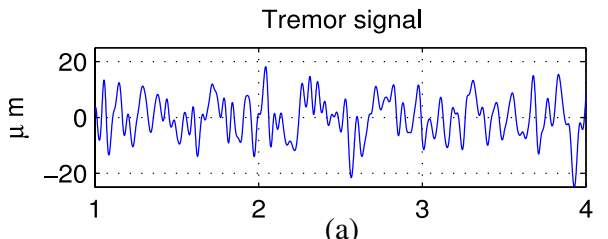

(a)

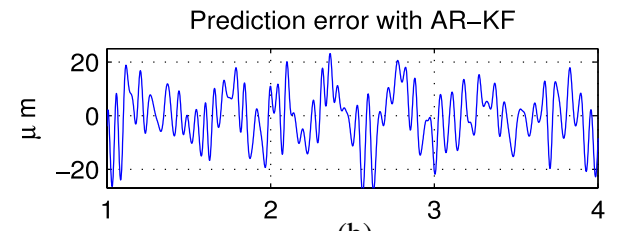

(b)
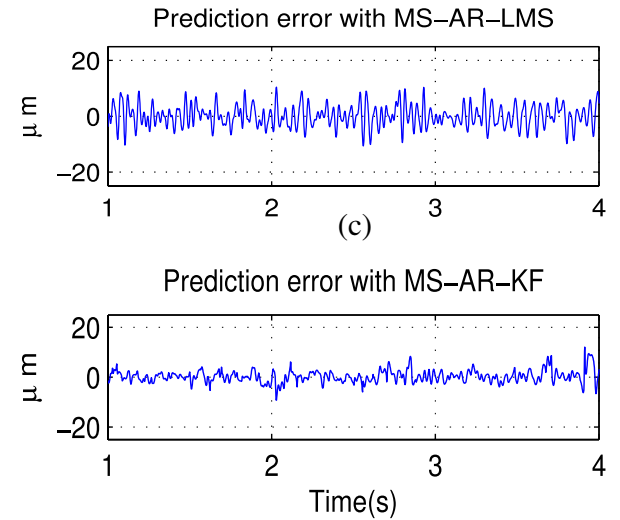

(d)

Fig. 11. Experimental results. (a) Tremor signal (Subject \#1, tracing task). (b) Prediction error with AR-KF. (c) Prediction error with MS-AR-LMS. (d) Prediction error with MS-AR-KF.

filter introduces delay, it is also required to remove the drift caused by the double integration of acceleration data to obtain position. The bandpass filter serves for dual purpose in eliminating the voluntary motion and the integration drift. Recently, in [6], a filter based on the ARMA model stochastic framework was proposed for online filtering of the pathological tremor and voluntary motion. Indeed, physiological and pathological tremors have different etiology and exhibit different characteristics in both time and spectral domains [37]. So, the filter in [6] is not suitable for the physiological tremor with multiple dominant frequency components.

BMFLC can filter the voluntary motion and tremor motion without the need of bandpass filtering [7], whereas AR methods 
and WFLC require an additional bandpass filtering stage (as performed in this paper) for the removal of the voluntary motion in the tremor signal. Further, these methods are not suitable when accelerometers are involved for sensing position as double integration is required. Comparatively, BMFLC can separate the voluntary and involuntary motion and can provide the tremor signal in the displacement domain without the need of numerical integration [7].

Microsurgery involves a lot of complex gestures, e.g., a intentional sudden jerk caused by a surgeon is a huge challenge for identification and filtering in real time. Moreover, when accelerometers are employed, unwanted drift is introduced due to numerical integration. Although BMFLC does not require the bandpass filter, due to presence of these high-frequency components and noise, a bandpass filter stage is always required when accelerometers are employed. Furthermore, for applications where tremor characteristics are not known, BMFLC requires a large bandwidth (e.g., 2-20 Hz bandwidth as employed in this paper) and this highly increases the computational complexity and affects the performance of BMFLC. Moreover, the bandpass filter stage can filter the tremor within a small bandwidth and it improves the filtering accuracy of BMFLC- and AR-based methods.

For the case of surgical devices, BMFLC-KF or AR-KF can both be employed for tremor prediction. However, for applications that requires onboard tremor estimation, with limited memory and processing speed, computational complexity can severely affect the performance. For those kinds of applications, AR-KF is more suitable.

For most of the subjects, 18-20 ms delay was identified as most surgeons tremor is in the high-frequency range. Although the phase delay caused by the bandpass filter can be time varying, an approximate constant phase lag can be estimated. Based on this lag, the multistep prediction was employed. For the case of time-varying delay, an adaptive multistep method that adjusts to the time delay can also be implemented. This issue will be discussed elsewhere.

\section{CONCLUSION}

In this paper, multistep prediction with BMFLC- and ARbased methods are developed for real-time tremor estimation for active cancellation. The analysis conducted for multistep prediction revealed that it largely improves the prediction accuracy. Comparatively, KF-based methods performed better compared to their LMS-based counterparts. WFLC-based methods are not suitable for multistep prediction due to the presence of single dominant frequency in the signal model. Both AR-KF and BMFLC-KF show similar performance and are more suitable for the tremor applications. Results with various prediction lengths and sampling frequencies highlight the robustness of the proposed approach. With an accurate estimation of delay, good estimation accuracy can be obtained for real-time applications.

\section{REFERENCES}

[1] S. G. Yuen, D. T. Kettler, P. M. Novotny, R. D. Plowes, and R. D. Howe, "Robotic motion compensation for beating heart intracardiac surgery," Int. J. Robot. Res., vol. 28, pp. 1355-1372, 2009.
[2] J. R. S. Koste, J. P. Cuijpers, F. G. Geest, F. J. Lagerwaard, B. J. Slotman, and S. Senan, "Verifying $4 \mathrm{~d}$ gated radiotheraphy using time-integrated electronic portal imaging: A phantom and clinical study," Radiat. Oncol., vol. 2, pp. 1-9, 2007.

[3] C. N. Riviere, J. Gangloff, and M. Mathelin, "Robotic compensation of biological motion to enhance surgical accuracy," Proc. IEEE, vol. 94, no. 9, pp. 1706-1716, Sep. 2006.

[4] G. P. Moustris, S. C. Hiridis, K. M. Deliparaschos, and K. M. Konstantinidis, "Evolution of autonomous and semi-autonomous robotic surgical systems: A review of the literature," Int. J. Med. Robot. Comput. Assist. Surg., vol. 7, pp. 375-392, 2011.

[5] T. Ortmaier, M. Groger, D. H. Boehm, V. Falk, and G. Hirzinger, "Motion estimation in beating heart surgery," IEEE Trans. Biomed. Eng., vol. 52, no. 10 , pp. $1729-1740$, Oct. 2005.

[6] A. P. L. Bo, P. Poignet, and C. Geny, "Pathological tremor and voluntary motion modeling and online estimation for active compensation," IEEE Trans. Neural Syst. Rehabil. Eng., vol. 19, no. 2, pp. 177-185, Apr. 2011

[7] K. C. Veluvolu and W. T. Ang, "Estimation of physiological tremor from accelerometers for real-time applications," Sensors, vol. 11, pp. 30203036, 2011.

[8] A. Ghorbanian, M. Zareinejad, S. M. Rezaei, H. Sheikhzadeh, and K. Bahestan, "A novel control architecture for physiological tremor compensation in teleoperated systems," Int. J. Med. Robot. Comput. Assist. Surg., 2012. Available: http://onlinelibrary.wiley.com/doi/10.1002/rcs.1436/abstract

[9] W. T. Ang, C. N. Riviere, and P. K. Khosla, "An active hand-held instrument for enhanced microsurgical accuracy," Med. Image. Comput. Comput. Assist. Interv., vol. 1935, pp. 878-886, 2000.

[10] W. T. Latt, U. X. Tan, C. Y. Shee, C. N. Riviere, and W. T. Ang, "Compact sensing design of a handheld active tremor compenstaion instrument," IEEE Sens. J., vol. 9, no. 12, pp. 1864-1871, Dec. 2009.

[11] R. J. Elbe and W. C. Koller, Tremor. U. MD, Ed. Baltimore, MD, USA: John Hopkins Univ. Press, 1985.

[12] G. Deuschl, J. Raethjen, and M. Lindemann, "The pathophysiology of tremor," Muscle Nerve, vol. 24, pp. 716-735, 2001.

[13] S. Charles, "Computer integrated surgery: Technology and clinical applications," in Dexterity Enhancement for Surgery, R. H. Taylor, Ed. Cambridge, MA, USA: MIT Press, 1996.

[14] C. N. Riviere and W. T. Ang, and K. P. K., "Toward active tremor canceling in handheld microsurgical instruments," IEEE Trans. Robot. Autom., vol. 19 , no. 5, pp. 793-800, Oct. 2003.

[15] J. A. Gallego, E. Rocon, J. O. Roa, J. C. Moreno, and J. L. Pons, "Realtime estimation of pathological tremor parameters from gyroscope data," Sensors, vol. 10, pp. 2129-2149, 2010.

[16] B. Carignan, J. F. Daneault, and C. Duval, "The organization of upper limb physiological tremor," Eur. J. Appl. Physiol., vol. 112, pp. 12691284, 2012.

[17] J. G. Gonzalez, E. A. Heredia, T. Rahman, K. E. Barner, and G. R. Arce, "Optimal digital filtering for tremor suppression," IEEE Trans. Biomed. Eng., vol. 47, no. 5, pp. 664-673, May 2000.

[18] W. T. Latt, K. C. Veluvolu, and W. T. Ang, "Drift-free position estimation of periodic or quasi-periodic motion using inertial sensors," Sensors, vol. 11, pp. 5931-5951, May 2011.

[19] A. V. Oppenheim and R. W. Schaefer, Discrete-Time Signal Processing Bergen Country, NJ, USA: Prentice-Hall, 2001

[20] H. N. Jacobus, A. J. Riggis, C. J. Jacobus, and Y. Weinstein, "Implementation issues for telerobotic handcontrollers: Human-robot ergonomice," in Human Robot Interaction, M. Rahimi and W. Karwowski, Eds. London, U.K.: Taylor \& Francis, 1992.

[21] J. Zhang and F. Chu, "Real-time modeling and prediction of physiological hand tremor," in Proc. IEEE 30th Annu. Int. Conf. Eng. Acoust., Speech, Signal Process., 2005, pp. 645-648.

[22] B. C. Becker, H. Tummala, and C. N. Riviere, "Autoregressive modeling of physiological tremor under microsurgical conditions," in Proc. IEEE 30th Annu. Int. Conf. Eng. Med. Biol. Soc., Vancouver, Canada, 2008, pp. 1948-1951.

[23] A. P. L. Bo, P. Poignet, F. Widjaja, and W. T. Ang, "Online pathological tremor characterization using extended Kalman filtering," in Proc. IEEE 30th Annu. Int. Conf. Eng. Med. Biol. Soc., Vancouver, Canada, 2008, pp. 1753-1756.

[24] G. Favier and D. Dubois, "A review of k-step-ahead predictors," Automatica, vol. 26, pp. 75-84, 1990.

[25] S. M. Hong, B. H. Jung, and D. Ruan, "Real-time prediction of respiratory motion based on a local dynamic model in an augmented space," Phys. Med. Biol., vol. 56, pp. 1775-1789, 2011.

[26] J. G. D. Gooijer and R. J. Hyndman, "25 years of time series forecasting," Int. J. Forecasting., vol. 22, pp. 443-473, 2006. 
[27] H. Cheng, P. N. Tan, J. Gao, and J. Scripps, "Multistep-ahead time series prediction," Lecture Notes Comput. Sci., vol. 3918, pp. 765-774, 2006.

[28] W. T. Latt, U. X. Tan, A. Georgiou, A. E. Sidarta, C. N. Riviere, and W. T. Ang, "A micro motion sensing system for micromanipulation tasks," Sens. Actuat. A-Phys., vol. 173, pp. 254-266, 2012.

[29] L. M. S. Eileen, W. T. Latt, W. T. Ang, T. C. Lim, C. L. Teo, and E. Burdet, "Micromanipulation accuracy in pointing and tracing investigated with a contact-free measurement system," in Proc. IEEE 31st Annu. Int. Conf. Eng. Med. Biol. Soc., Minneapolis, MN, USA, 2009, pp. 3960-3963.

[30] Y. K. Thong, M. Woolfson, J. A. Crowe, B. R. Hayes-Gill, and D. A. Jones, "Numerical double integration of acceleration measurements in noise," Measurement, vol. 36, no. 1, pp. 73-92, Jul. 2004.

[31] S. Haykin, Adaptive Filter Theory. Englewood Cliffs, NJ, USA: Prentice-Hall, 2001.

[32] B. Widrow and S. D. Stearns, Adaptive Signal Processing. Englewood Cliffs, NJ, USA: Prentice-Hall, 1985.

[33] M. P. Tarvainen, J. K. Hiltunen, P. O. Ranta-aho, and P. A. Karjalainen, "Estimation of nonstationary EEG with Kalman smoother approach: An application to event-related synchronization (ERS)," IEEE Trans. Biomed. Eng., vol. 51, no. 3, pp. 516-524, Mar. 2004.

[34] H. Akaike, "A new look at the statistical model identification," IEEE Trans. Autom. Control., vol. 19, no. 6, pp. 716-723, Dec. 1974.

[35] H. Das, H. Zak, J. Johnson, J. Crouch, and D. Frambach,"Evaluation of a telerobotic system to assist surgeons in microsurgery," Comput. Aided Surg., vol. 4, pp. 15-25, 1999.

[36] K. C. Veluvolu, W. T. Latt, and W. T. Ang, "Double adaptive bandlimited multiple fourier linear combiner for real-time estimation/filtering of physiological tremor," Biomed. Signal Process. Control., vol. 5, pp. 37-44, Jan. 2010.

[37] J. Timmer, C. Gantert, G. Deuschl, and J. Honerkamp, "Characteristics of hand tremor time series," Biol. Cybern., vol. 70, pp. 75-80, May 1993.

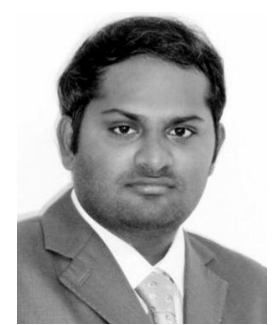

Kalyana C. Veluvolu (S'03-M'06-SM'13) received the B.Tech. degree in electrical and electronic engineering from Acharya Nagarjuna University, Guntur, India, in 2002, and the Ph.D. degree in electrical engineering from Nanyang Technological University, Singapore, in 2006.

Since 2009, he has been with the College of IT Engineering, Kyungpook National University, Daegu, Korea, where he is currently an Associate Professor. During 2006-2009, he was a Research Fellow in Biorobotics Group, Robotics Research Center, Nanyang Technological University. His current research interests include nonlinear estimation and filtering, sliding mode control, brain-computer interface, biomedical signal processing, and surgical robotics.

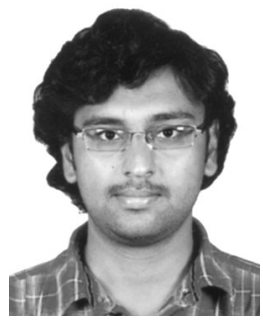

Sivanagaraja Tatinati received the B.Tech. degree in electronics and communication engineering from Acharya Nagarjuna University, Guntur, India, in 2010. He is currently working toward the Ph.D degree at Kyungpook National University, Daegu, Korea.

His current research interests include robotics assisted medical instruments, adaptive filtering, application of control, and estimation algorithms in biomedical engineering.

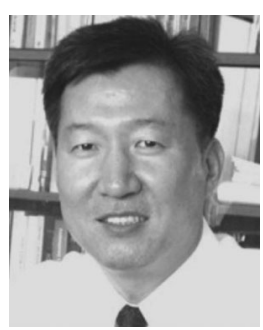

Sun-Mog Hong (S'86-M'89) received the B.S. degree from Korea Aerospace University, Seoul, Korea, in 1978, and the M.S. degree from the Korea Advanced Institute of Science and Technology, Daejon, Korea, in 1980, both in electronic engineering, and the M.S. and Ph.D. degrees in aerospace engineering from the University of Michigan, Ann Arbor, MI, USA, in 1985 and 1989, respectively.

Since 1980, he has been with the School of Electronics Engineering, Kyungpook National University, Daegu, Korea, where he is currently a Professor. His current research interests include motion prediction for robotic surgical systems and target tracking/track fusion in radar networks.

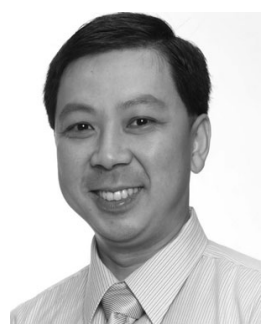

Wei Tech Ang (S'98-M'04) received the B.E. and M.E. degrees in mechanical and production engineering from Nanyang Technological University, Singapore, in 1997 and 1999, respectively, and the Ph.D. degree in robotics from Carnegie Mellon University, Pittsburgh, PA, USA, in 2004.

Since 2004, he has been with the School of Mechanical and Aerospace Engineering, Nanyang Technological University, where he is currently an Associate Professor and holds the appointment of Head of Engineering Mechanics Division. His research focuses on robotics technology for Biomedical applications, which include surgery, rehabilitation and cell micromanipulation. 